TRANSGENESIS

\title{
Mosquitoes bite back on parasite transmission
}

Mosquito bites aren't just annoying: they are also often deadly, as mosquitoes are the obligate vectors for the malarial parasite Plasmodium. One way to reduce disease transmission by mosquito bites might be to genetically engineer the mosquitoes themselves, as a new study published by Junitsu Ito and colleagues in Nature suggests. Plasmodium enters the mosquito in an infected blood meal. It then proliferates in the midgut of the mosquito and is transmitted to a new host through another bite. After entering the host, the parasite must move through several mosquito epithelia to complete its development, thus providing a possible target to stop its deadly life cycle.

In previous work, the same lab identified a peptide that binds to mosquito epithelia. An excess of peptide can swamp out parasite-epithelial interactions, thus preventing the parasite from developing in the mosquito. The new work has taken this principle one step further with the creation of transgenic mosquitoes that express four linked copies of this peptide behind a signal peptide. Importantly,

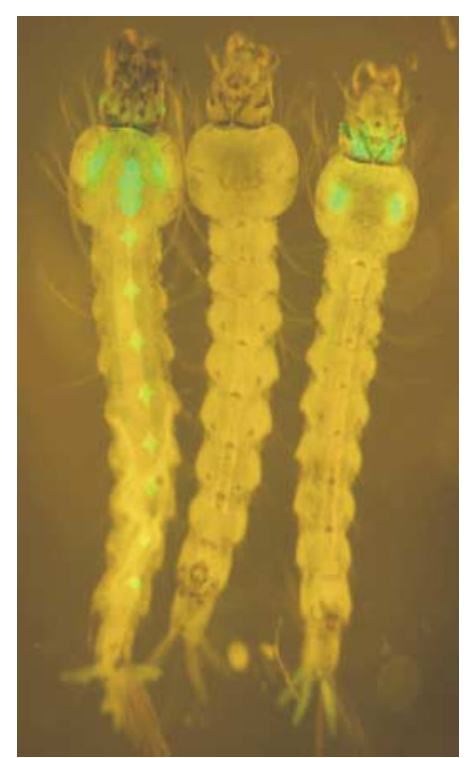

Transgenic mosquito larvae, as shown by green fluorescence (left and right). Photo courtesy of Junitsu lto et al. Nature ( $)$ (2002) Macmillan Magazines Ltd. this multi-peptide protein is expressed from a promoter that is turned on in the midgut, specifically when the mosquito feeds on blood. In this way, the peptide is expressed in the right place and at the right time to interfere with the parasite's life cycle. The transgenic mosquitoes (see accompanying picture) had a significantly lower susceptibility to parasite infection. Crucially, the authors then showed that parasite transmission levels were lowered by half in one transgenic line, and in two lines they were lowered to undetectable levels.

The possibility of stopping malarial transmission is an exciting one; however, as often with a milestone paper on a novel application, there are some problems to be surmounted. Plasmodium, like many organisms, has considerable genetic diversity and so might quickly develop resistance to the transgenic mosquitoes by using a different method to stay alive in the host. Thus, Ito et al. are continuing to look for additional genes that can be used in conjunction with the one encoding the peptide to completely stop transmission. The imminent publication of the genomes of both the parasite Plasmodium falciparum and the mosquito strain Anopheles gambiae should, of course, accelerate this research. However, even when mosquitoes are generated with several genetic modifications, there remain numerous problems with dispersing these mosquitoes in the field, and much thought will be needed before introducing these modifications into the environment. Yet, the prospect of preventing a disease that kills millions of humans every year is certainly strong motivation to explore all available options.

Chris Gunter, Associate Editor, Nature (4) References and links ORIGINAL RESEARCH PAPER Ito, J. et al. Transgenic anopheline mosquitoes impaired in the transmission of a malaria parasite. Nature $\mathbf{4 1 7}$ 452-455 (2002)

\section{WEB SITE}

Marcelo Jacobs-Lorena's faculty page: http://genetics.cwru.edu/jacobslorena.htm

\section{IN BRIEF}

\section{TECHNOLOGY}

Targeted mutagenesis by homologous recombination in Drosophila melanogaster.

Rong, Y. S. et al. Genes Dev. 16, 1568-1581 (2002)

Gene targeting by homologous recombination is a recent addition to the fly genetic toolkit. The lab that first described the technique two years ago now successfully addresses its general utility. By using a modified version of the original methods, the authors have generated specific alleles of five genes, using only knowledge of the DNA sequence of the targeted locus. Insertional mutations and allelic substitutions can be easily generated with this technique, except in telomeric regions.

\section{DEVELOPMENT}

Neurogenin 3 is essential for the proper specification of gastric enteroendocrine cells and the maintenance of gastric epithelial identity.

Lee, C. S. et al. Genes Dev. 16, 1488-1497 (2002)

Downstream targets of activated Notch - the proneural genes, such as neurogenin 3, math 1 and Neurod - have been shown to drive differentiation of endocrine cells in the gut epithelium. Lee et al. extend these findings by constructing an EGFP-marked null allele of neurogenin 3 . They show that the gene is required for enteroendocrine cell differentiation in the stomach and for the maintenance of gastric epithelial identity.

\section{CANCER GENETICS}

\section{Mutations of the BRAF gene in human cancer.}

Davies, H. et al. Nature 9 June 2002 (10.1038/nature00766)

This is the first report from a systematic genome-wide screen for genes that are frequently mutated in cancers. The authors compared genomic DNA from 15 cancer cell lines with normal lymphoblastoid cell lines from the same individuals, focusing on the region around $B R A F$ - a downstream target of RAS. This, and additional analyses, identified mutations in BRAF that associate with cancers, especially with malignant melanomas. Of such melanomas, $66 \%$ are positive for a missense BRAF mutation that constitutively stimulates the RAS pathway.

\section{GENE THERAPY}

\section{Restoration of spermatogenesis by lentiviral gene} transfer: offspring from infertile mice.

Ikawa, M. et al. Proc. Natl Acad. Sci. USA 99, 7524-7529 (2002)

Mice that are mutant for Steel (Sl), which encodes the ligand (Kitl) for the c-kit receptor, are good models for human male infertility, as Kitl is required in Sertoli cells to support normal spermatogenesis. Lentiviral-mediated transfer of functional Kitl to the Sertoli cells of $S l / S l^{d}$ mice rescued spermatogenesis defects and infertility in all testes studied. None of the sired pups carried the transgene, making lentiviral transmission a possible means to treat human male infertility. 